\title{
Continuity as a Galaxy of Hyperreal Functions
}

\author{
Ibrahim O. Hamad \\ ibrahim.hamad@su.edu.krd \\ College of Sciences \\ University-Salahaddin
}

Received on: 19/8/2007

Accepted on: 4/11/2007

\begin{abstract}
In the present paper, the problem of defining continuity and scontinuity as a galaxy of hyperreal function is discussed. Our attempt is based on the fact that monads are subsets of some galaxies. New results are obtained, with nonstandard variables, related to a new extension of the continuity notion.

Keywords: continuity, s- continuity, monads, galaxy.$$
\text { الاستمرارية كمجرة لاوال حقيقية فوقية }
$$

$$
\begin{aligned}
& \text { إبراهيم عثمان حمد } \\
& \text { كلية العلوم } \\
& \text { جامعة صلاح الدين }
\end{aligned}
$$

تاريخ قبول البحث: 2007/11/4

تاريخ استلام البحث: 2007/8/19

(الملخص

في هذا البحث تم تتاول مسالة تعريف الاستمرارية و س - الاستمرارية كمرة لدوال

الحقيقية الفوقية. محاولتنا بنيت على حقيقة كون موناد هو مجموعة جزئية من بعض المجرات، وقد

حصلنا على نتائج جديدة في دوال المعرفة على منطلقات غير قياسية وتم تعميم مفهوم الاستمرارية

ليشمل جميع حالات الاستمرارية لدالة معرفة على منطلقات قياسية وغير قياسية.

الكلمات المفتاحية: استمرارية، س-استمرارية، هالة، مجرة.
\end{abstract}

\section{1- Introduction: -}

The following definitions of nonstandard analysis will be needed in this paper.

Every set or elements defined in a classical mathematics is called a standard. [2] 
Any set or formula which does not involve a new predicates "standard, infinitesimals, limited, unlimited...etc" is called internal, otherwise is called external. [2] ,[4]

A real number $x$ is called unlimited if $|x|>r$ for all real $r>0$ otherwise called limited.[2]

The set of all unlimited real numbers is denoted by $\overline{\mathbf{R}}$, and the set of all limited real numbers is denoted by $\underline{\mathbf{R}}$ [2]

A real number $x$ is called infinitesimal if $|x|<r$ for all positive standard real number $r$ [4].

A real number $x$ is called appreciable if it is neither unlimited nor infinitesimal, and the set of all positive appreciable numbers is denoted by $\mathrm{A}^{+} \cdot[4]$

Two real numbers $x$ and $y$ are said to be infinitely close if $x-y$ is infinitesimal and is denoted by $x \cong y$.[4]

If $x$ is a limited number in $\mathbf{R}$, then it is infinitely close to a unique standard real number, this unique number is called the standard part of $x$ or shadow of $x$ denoted by $s t(x)$ or ${ }^{0} x$, in this case we say that $x$ is nearly standard [2][6].

Assume that $f$ is a real valued function then:

1- A standard function $f$ is continuous at a standard point $x_{o}$ if for all $x$ $x \cong x_{o}$ then $f(x) \cong f\left(x_{o}\right) \cdot[4]$ 2- $f$ is called s-continuous at $x_{0}$ if for all $x, x \cong x_{0}$ then $f(x) \cong\left(x_{0}\right)$.

A standard function is bounded or limited if there exists a standard real constant number $k$ such that $|f(x)|<k$.

$\operatorname{monad}(x)=\{y \in \mathbf{R}: x \cong y\}$ for limited $x$, and is denoted by $m(x) .[2]$

$\alpha-\operatorname{monad}(x)=\left\{y \in \mathbf{R}: \frac{y-x}{\alpha} \cong 0\right\}$, and is denoted by $\alpha-m(x) \cdot[2]$

$\alpha-\operatorname{micromonad}(x)=\left\{y: y-x<\varepsilon^{n} \quad \forall\right.$ standadd $\left.n\right\}$ [2]

$\operatorname{galaxy}(x)=\{y \in \mathbf{R}: y-x \quad$ limited $\}$ and is denoted by $\operatorname{gal}(x)$ [2]

$\alpha-\operatorname{galaxy}(x)=\left\{y \in \mathbf{R}: \frac{y-x}{\alpha}\right.$ limited $\}$ [2] 
A function $f: A \rightarrow B$ is said to be internal if $A$ is internal [1].

Let $f$ be an internal function, we say that $f$ satisfies $\boldsymbol{k}$-Lipschitz condition if $|f(x)-f(y)|<k|x-y|$ for some standard constant $k \in \mathbf{N}$.[5]

If $E$ is a standard metric space, then $E$ is complete if for each $x \in E$ either $x$ is nearly standard or there exists a standard number $r>0$, such that the ball $B(x, r)$ contains no standard elements. [2]

\section{Remark 1.1:}

1. From the definition of monad and galaxy we have: $\forall t \in \mathbf{R}, \quad m(t) \subset \operatorname{gal}(t)$.

2. Using the definition of monad we obtain:

A standard function $f$ is continuous at a standard point $x_{o}$ if for all $x, x \in m\left(x_{0}\right)$ then $f(x) \in m\left(f\left(x_{0}\right)\right)$.

\section{2- Galaxy Continuity}

With nonstandard analysis the region of tangible elements is larger than that of standard analysis, therefore all problems that deal with such unusual elements take its frame space in nonstandard analysis, so the study of the behaviour of a function and its properties in nonstandard analysis give us a real phase and precision of the nature of it, which can never be imagined classically.

For functions that deal with only unlimited values or its values does not lie in the monad of any other points, the conventional and nonstandard definitions of continuity are meaningless and we are unable to say any thing about its continuity. In this paper we treat such problem by presenting a new version of continuity which includes all possible standard and nonstandard cases.

\section{Definition 2.1:}

Let $f: X \rightarrow Y$ be an internal function then we say that $f$ is galaxicaly continuous at a point $x_{0} \in X$ if for all $x, x \in m\left(x_{0}\right)$ then $f(x) \in \operatorname{gal}\left(f\left(x_{0}\right)\right)$, and is denoted by g-continuous.

The following examples give some relations between continuity, scontinuity, and g-continuity.

\section{Examples 2.2}

i) Let $f(x)=\frac{1}{x} \quad x \neq 0$, then we have: 
1- For all standard $x, f$ is continuous, and neither s-continuous nor g-continuous.

2- If $x \cong x_{o}$ such that $x \in x \cdot x_{o}-\operatorname{gal}\left(x_{o}\right)$, then $f$ is gcontinuous at $x_{o}$, and otherwise it is not g-continuous.

3- $f$ is not s-continuous, take $x_{0}=\frac{1}{\omega}$, and $x=\frac{1}{a \omega}$ where $\omega$ is unlimited and $a>1$ is a standard real number we have $x \in m\left(x_{0}\right)$ while $f(x) \notin m\left(f\left(x_{0}\right)\right)$ also $f(x) \notin g a l\left(f\left(x_{0}\right)\right)$.

ii) $f(x)=\left\{\begin{array}{cc}\sin \left(\frac{1}{x}\right) & x \in(-\varepsilon, 0) \cup(0, \varepsilon) \\ 0 & x=0\end{array}\right.$

where $\varepsilon \cong 0$,

is not continuous at 0 but it is g-continuous.

iii) $f(x)= \begin{cases}1 & x \in \mathbf{Q} \text { is not continuous at any real point while it } \\ 0 & x \notin \mathbf{Q}\end{cases}$ is g-continuous everywhere.

iv) $f(x)=e^{\frac{x}{\varepsilon}}, x \in[0, n \varepsilon]$, where $n$ is standard positive integer, and $\varepsilon \cong 0$ is $\quad$ g-continuous but not s-continuous.

\section{3- Main results}

\section{Theorem 3.1}

1. Let $f$, and $x_{o}$ be standard, if $f$ is continuous at $x_{o}$ then $f$ is g-continuous at $x_{o}$.

2. If $f$ is s-continuous then $f$ is g-continuous.

3. If $f$ satisfies $\boldsymbol{k}$-Lipschitz condition then $f$ is g-continuous.

\section{Proof:}

1. Let $f$ be a standard continuous function at a standard point $x_{o}$ then from the definition of continuity we have:

for all $x \quad x \cong x_{o}$ then $f(x) \cong f\left(x_{o}\right)$,

use definition of monad we get

for all $x, x \in m\left(x_{0}\right)$ then $f(x) \in m\left(f\left(x_{0}\right)\right)$. 
Since $\forall t \in \mathbf{R}, \quad m(t) \subset \operatorname{gal}(t)$, then we get:

for all if for all $x, x \in m\left(x_{0}\right)$ then $f(x) \in \operatorname{gal}\left(f\left(x_{o}\right)\right)$,

thus $f$ is g-continuous.

2. It's proof is similar to the proof of (1).

3. Let $f$ be an internal function (standard or nonstandard) such that $f$ satisfies $\boldsymbol{k}$-Lipschitz condition and $x, y \in \mathbf{R}$ such that $x \cong y$, to prove that $f(x) \in \operatorname{gal}(f(y))$,

Since $f$ satisfies $\boldsymbol{k}$-Lipschitz condition then we get

$|f(x)-f(y)|<k|x-y|$ for some standard constant $k \in \mathbf{N}$,

but $x \cong y$, so $|f(x)-f(y)|$ is limited,

therefore $f(x) \in \operatorname{gal}(f(y))$.

\section{Remark: -}

The converse of the above theorem is not true, for $\mathbf{1}$. and $\mathbf{2}$.see examples 2.2(iii) and 2.2(ii) respectively.

For the third statement let $f(x)=\left\{\begin{array}{ll}x & x>0 \\ 5 & x \leq 0\end{array}\right.$, its clear that $f$ is g-continuous and to show that it does not satisfy $\boldsymbol{k}$-Lipschitz condition;

Let $x=0$ and $y=\varepsilon$ where $\varepsilon$ is an infinitesimal number, then

$x \cong y$ and $|x-y| \cong 0$, but there is no standard number $k$ satisfies the inequality $|f(x)-f(y)|<k|x-y|$.

\section{Theorem 3.2}

Let $f(x) \in \varepsilon-\operatorname{gal}(f(y))$ for all $x \cong y$, where $\varepsilon$ is an infinitesimal number, then $f$ is g-continuous if and only if $f$ is uniformly s- continuous.

\section{Proof:}

let $f$ be g-continuous, then foe all $x, x \cong y$ we have

$$
f(x) \in \operatorname{gal}(f(y)) .
$$

That is

$$
f(x)-f(y) \text { is limited. }
$$

Since

$$
f(x) \in \varepsilon-\operatorname{gal}(f(y)),
$$

then

$$
\frac{f(x)-f(y)}{\varepsilon} \text { is limited }
$$

That is $\quad f(x)-f(y)=k \varepsilon^{n}$ for some standard numbers $k$ and $n$. 
Thus $\quad f(x)-f(y)$ is infinitesimal

Therefore $\quad f$ is uniformly s- continuous.

The converse is obvious.

\section{Theorem 3.3}

Every limited function is uniform g-continuous.

\section{Proof:}

Assume that $f$ is a limited function

Let $x \cong y$, since $f$ is limited, then

$\forall x \in D_{f}$ we have $|f(x)|<k$ for some standard positive integer $k$.

Since

$$
|f(x)-f(y)| \leq|f(x)|+|f(y)|<2 k,
$$

So that $\quad f(x)-f(y)$ is limited.

That is $\quad f(x) \in \operatorname{gal}(f(y))$,

then $f$ is uniform g-continuous.

\section{Remark: -}

The converse of Theorem 3.3 is not true in general (remembering that classically every continuous functions defined on a closed interval is limited). See example 2.2(i) on the bounded interval $(0,1]$

\section{Corollary 3.4}

Let $f:[a, b] \rightarrow \mathbf{R}$ be a standard continuous function, and $h$ be a function such that $h(x)<f(x)$ for all $x, x \in[a, b]$, then $h$ is $\mathrm{g}$ continuous.

\section{Proof:}

Use Theorem 2.8 [3] to get that $f$ is limited.

Therefore $h$ is limited,

Now, use Theorem 3.3 to obtain the result.

\section{Theorem 3.5}

Let $f$ be an infinitesimal valued function, then $f$ is s-continuous function if and only if $f$ is g-continuous.

\section{Proof:}

The forward way is direct.

For the backward direction, let $f$ be g-continuous function,

Therefore $\quad f(x) \in \operatorname{gal}(f(y)) \forall x, x \cong y$, 
That is $\quad f(x)-f(y)$ is limited

but $\quad f(x) \cong 0$,

so $\forall x \in D_{f}$ we have $f(x)-f(y)$ is infinitesimal.

Thus $f$ is s-continuous.

\section{Theorem 3.6}

Let $f$ be an internal function, then $f$ is g-continuous if and only if $f(x) \in \alpha-\operatorname{gal}(f(y))$ for all $x, x \cong y$ and $\alpha$ is appreciable.

\section{Proof:}

Let $f$ be g-continuous, to prove that $f(x) \in \alpha-\operatorname{gal}(f(y))$ for all $x$, $x \cong y$, we have to prove that $\frac{f(x)-f(y)}{\alpha}$ is limited.

Since $f$ is g-continuous then, $f(x)-f(y)$ is limited so the result is obtained.

Conversely,

let $x \cong y$, since $f(x) \in \alpha-\operatorname{gal}(f(y))$ and $\alpha$ is appreciable,

Therefore

$$
\frac{f(x)-f(y)}{\alpha} \text { is limited, }
$$

Thus

$$
f(x)-f(y) \text { is limited, and then } f \text { is g-continuous. }
$$

\section{Theorem 3.7}

Let $X$ and $Y$ be two metric spaces, and $A$ be a complete subset of $X$. If $f$ is s-continuous function from $A^{c}$ into $Y$, then there exist a function $h: X \rightarrow Y$ which is g-continuous.

\section{Proof:}

Let $x \in X$,

if $x \in A^{c}$ then define $h(x)=f(x)$,

since s-continuity implies g-continuity, therefore $h$ is g-continuous.

Now, for every $x \in A$,

We have to find a function $h$ such that $h$ is a g-continuous function.

Since $A$ is complete then for all $x \in A$, 
either $x$ is nearly standard or there exists a standard $r>0$, such that the ball $B(x, r)$ contain no standard element.

In the first case, there exist ${ }^{o} x$ in $X$ such that $x \cong{ }^{o} x$.

Therefore, for all $x \in A$, define $h(x)={ }^{o} x$ and assume that $x \cong y$, then

$h(x)={ }^{o} x \cong x \cong y \cong{ }^{o} y=h(y)$.

Thus $h$ is g-continuous.

In the second case, there exists a standard $r>0$, such that for all $y \in B(x, r)$, then $y$ is not standard.

Therefore we have the following concerning the value of $y$ :

i. $y$ is infinitesimal.

ii. $y$ is appreciable.

iii. $y$ is unlimited.

For the first two possibilities define $h(x)={ }^{o} x$,

then $h(x)-h(y)$ is limited, therefore $h$ is g-continuous.

For the third possibility, if $x \cong y$, then $x$ is also an unlimited number, if $x \nsubseteq y$ we get a contradiction to our assumption.

In this case, define $h(x)=x+t_{x}$, where $t_{x}$ is any limited real number related with the variable $x$

Therefore

$$
\begin{gathered}
h(x)-h(y)=x-y+\left(t_{x}-t_{y}\right), \\
\cong\left(t_{x}-t_{y}\right),
\end{gathered}
$$

which is limited.

Thus, the function

$$
h(x)=\left\{\begin{array}{cc}
f(x) & \text { if } x \in A^{c} \\
{ }^{o} x & \text { if } x \text { is nearly standard } \\
x+t_{x} & \text { if } x \text { is is unlimited }
\end{array}\right.
$$

is g-continuous. 


\section{$\underline{\text { REFERENCES }}$}

[1] Davis, M. (1977): Applied Nonstandard Analysis, New York, John Wiley \& Sons.

[2] Diener, F.\& Diener, M. (1996): Nonstandard Analysis in Practice, Springer-Verlag, Berlin,Heildeberg.

[3] Ibrahim O. Hamad (2005): On Some Nonstandard Results of Continuous \& Monotonic Functions J.ED. \& Sc., Vol.17.

[4] Nelson, E. (1977): Internal set Theory, Bulletin of the American Mathematical Society, Vol.83,No.6,pp1165-1198.

[5] Pugh, C.C. (2002): Real Mathematical analysis, Springer- Verlag New York, Inc.

[6] Van Den Berg, I. (1984):Halos and ,Galaxies: Application on the Real Line, Serie de math. Pures et Appliquees, IRMA-Strasbourg. 